\title{
Harvesting the Thermal Cardiac Pulse Signal
}

\author{
Nanfei Sun ${ }^{1}$, Ioannis Pavlidis ${ }^{1, \star}$, Marc Garbey $^{2}$, and Jin Fei ${ }^{1}$ \\ ${ }^{1}$ Computational Physiology Lab \\ ${ }^{2}$ Department of Computer Science \\ University of Houston, Houston, TX, USA \\ \{nsun, jinfei, garbey\}@cs.uh.edu, \\ ipavlidi@central.uh.edu
}

\begin{abstract}
In the present paper, we propose a new pulse measurement methodology based on thermal imaging (contact-free). The method capitalizes both on the thermal undulation produced by the traveling pulse as well as the periodic expansion of the compliant vessel wall. The paper reports experiments on 34 subjects, where it compares the performance of the new pulse measurement method to the one we reported previously. The measurements were ground-truthed through a piezo-electric sensor. Statistical analysis reveals that the new imaging methodology is more accurate and robust than the previous one. Its performance becomes nearly perfect, when the vessel is not obstructed by a thick fat deposit.
\end{abstract}

\section{Introduction}

The research described in this paper aims to recover robustly the pulse frequency in a contact-free manner. This effort is part of a general framework that we established for measuring multiple vital signs. The hallmark of the framework is that all measurements are performed at a distance and under a single sensing regime (thermal imaging). So far we have demonstrated that it is possible to perform at a distance measurements of pulse [1, breathing rate 2, 3, superficial vessel blood flow [4, and perfusion [5. The technology is very appealing in the context of psycho-physiology, where outfitting the subject with contact sensors is not recommended. In psychological experiments it is very important for the subject to feel as free as possible or a variable may be introduced in his psychological state. As the technology for measuring vital signs at a distance matures, it may find biomedical applications beyond psycho-physiology, wherever sustained physiological measurements are of interest.

Cardiac pulse is an important vital sign that reflects the health status of the subject's cardiovascular system. It is also indicative of the metabolic rate and stress level of the subject. In [1 we introduced a thermal imaging method to measure pulse. That paper established the feasibility of measuring pulse at a distance using passive sensing. In the present manuscript we report substantial improvements that take the initial method from the realm of feasibility to the realm of applicability.

\footnotetext{
^ Corresponding author.
} 
In the rest of the paper we unveil our new imaging methodology for measuring pulse. Specifically, in section 2, we describe the method itself. In section 3, we report and discuss its experimental validation.

\section{Pulse Measurement Methodology}

\subsection{Cross-Section Temperature Function}

Within the rectangular region of interest, the operator draws a line that traverses the cross-section of the thermal imprint of the vessel (e.g., carotid). The line has to bleed over to the surrounding tissue (see Fig. 1). By applying our measurement methodology on this line over time, we can capture the thermal undulation caused by pulsative vessel blood flow. For the typical experimental configuration we use (subject at $6 \mathrm{ft}$ and camera outfitted with a $50 \mathrm{~mm}$ lens) the cross-section of a major vessel, such as the carotid, spans between

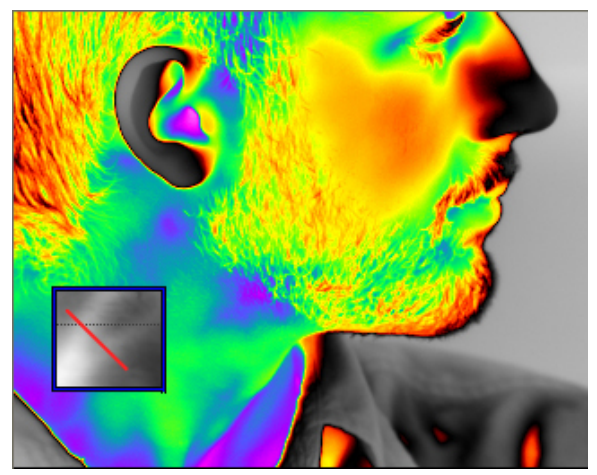

Fig. 1. Carotid region of interest and cross-sectional measurement line

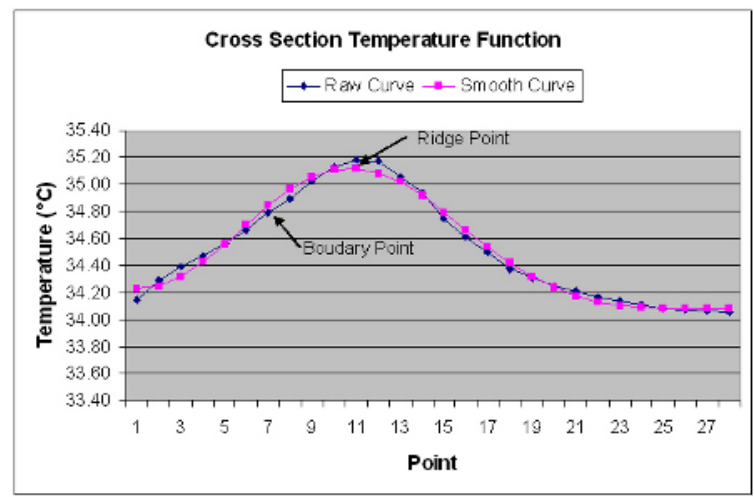

Fig. 2. Temperature profile across the vessel thermal imprint 
$5-10$ pixels. We increase the low spatial resolution of the measurement line by applying quadratic interpolation [6]. The points of the interpolated measurement line correspond to temperatures and form the cross-section temperature function $g_{t}(x), x \in\{-N, \ldots, N\}$ at frame $t$. We model the cross-section temperature function using the first five (5) cosine functions of the Fourier series 7 . The modeling yields a smoother curve $h_{t}(x), x \in\{-N, \ldots, N\}$ at frame $t$ (see Fig. 2).

\subsection{Ridge and Boundary Temperature Functions}

We use the facial tissue tracking algorithm we reported in 8 to compensate for natural head motion during measurement. This is not sufficient, however, since there is fine vessel displacement not related to volitional head movement. This displacement is due to minute motor motion, diaphragm induced motion, and the elastic response of the vessel to systolic and diastolic pressure. The vessel displacement moves the point of the maximum temperature reading along the measurement line. We call this point, ridge point and it corresponds to the middle of the vessel's cross section, where the blood flow speed is maximal. At frame $t$, the ridge point is defined as:

$$
r_{t}=\arg \max _{x}\left\{h_{t}(x)\right\}, x \in\{-N, \ldots, N\},
$$

where $h_{t}(x)$ is the smoothed cross-section temperature function. The time evolution of the ridge point forms the ridge line, which is indicative of the vessel's displacement. The ridge line maps to the ridge temperature function $l_{r}(x, t), x \in$ $\{-N, \ldots, N\}, t \in\{1, \ldots, T\}$, which is an accurate record of the evolution of the vessel's maximum temperature (strong signal).

The vessel's minimum temperature is recorded at the vessel's boundary, where the blood flow speed is minimal. At each frame $t$, we select on either side of the measurement line, the boundary point $b_{t}$ to be:

$$
b_{t}=\arg \max _{x}\left\{\left|h_{t}^{\prime}(x)\right|+\left|h_{t}^{\prime \prime}(x)\right|\right\}, x \in\{0, \ldots, N\},
$$

where $h_{t}(x)$ is the smoothed cross-section temperature function. The time evolution of the boundary point forms the boundary line. The boundary line does not exactly mirror the displacement of the ridge line. The reason is that the vessel is compliant and its volume changes with respect to pressure. Therefore, the vessel expands during diastole and contracts during systole, superimposing a boundary deformation on the general vessel displacement. The boundary line maps to the boundary temperature function $l_{b}(x, t), x \in\{0, \ldots, N\}, t \in\{1, \ldots, T\}$, which is an accurate record of the evolution of the vessel's minimum temperature. This function carries valuable pulse information that is associated to the periodic expansion of the vessel's wall. Fig. 3 depicts the ridge and boundary lines and the corresponding temperature functions for a measurement applied on the carotid of a subject. The measurement lasted for $T=1,000$ frames. 


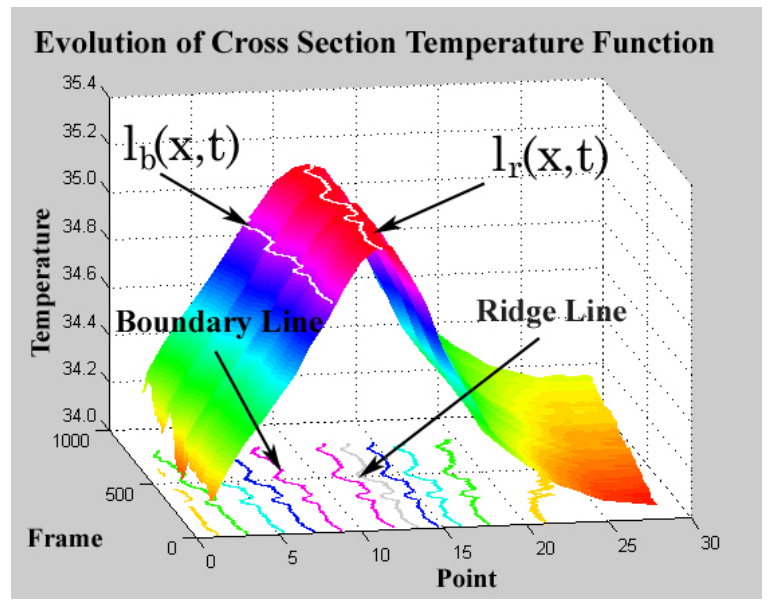

Fig. 3. Ridge and boundary lines along with the corresponding temperature functions

\subsection{Computation of the Mean Pulse Frequency}

Initially, we compute the mean pulse over an extended period of time $T$ ( $T \geq$ $30 \mathrm{sec}$ ). In long observation periods the pulse frequency is expected to dominate in the spectral domain, since it is more consistent than white noise. Therefore, this reliable mean value estimate can be used in a feedback loop to narrow-down the search space in a second-pass instantaneous pulse computation.

The computation is based on Fourier analysis and takes into account both the ridge and boundary temperature functions $l_{r}(x, t)$ and $l_{b}(x, t)$ respectively. We will show the development for the ridge temperature function $l_{r}(x, t)$ only. Exactly, the same applies for the boundary function $l_{b}(x, t)$. Specifically:

1. We use a low order trigonometric polynomial to prepare the function $l_{b}(x, t)$ for Fast Fourier Transformation (FFT):

$$
L_{r}(x, t)=l_{r}(x, t)-(\alpha \cos (t)+\beta),
$$

where $\alpha=\frac{1}{2}\left(L_{r}(x, 0)-L_{r}(x, T-1)\right)$ and $\beta=\frac{1}{2}\left(L_{r}(x, 0)+L_{r}(x, T-1)\right)$. This ensures that the shift will not affect the stability of the scheme by minimizing the Gibbs phenomenon.

2. We extend $L_{r}(x, t)$ to a $2 T$ periodic function as follows: we apply a symmetry function (Eq. (4)) and then a periodic extension (Eq. (5D):

$$
\begin{gathered}
\forall t \in(0, T), L_{r}(x, T-t)=-L_{r}(x, t) \\
\forall t \in(0,2 T), \forall k \in Z, L_{r}(x, t+k 2 T)=L_{r}(x, t)
\end{gathered}
$$

3. We apply a classic decimation-in-time (Cooley and Tukey) $1 \mathrm{D}$ base-2 FFT method [6] to obtain the power spectrum $P_{r}(f)$ of function $L_{r}(x, t)$ :

$$
P_{r}(f)=\mathcal{F}\left(L_{r}(x, t)\right) .
$$


4. We model the power spectrum $P_{r}(f)$ as a multi-Normal distribution $P_{r}^{\prime}(f)$ by applying a Parzen window method [9]:

$$
P_{r}^{\prime}(x)=\frac{1}{F} \sum_{i=1}^{F} W\left(f-f_{i}\right),
$$

where $W(f)$ is the Parzen window Normal kernel:

$$
W(x)=\frac{1}{\sigma_{p} \sqrt{2 \pi}} e^{-\frac{\left(x-\mu_{p}\right)^{2}}{2 \sigma_{p}^{2}}} .
$$

We take as $\mu_{p}=0$ and $\sigma_{p}^{2}=0.1$. The normalized mean frequency variance of the pulse for the subjects in our data set is $\overline{\sigma_{n}^{2}}=0.1$, as it is computed from the ground-truth measurements. Therefore, our choice $\sigma_{p}^{2}=0.1$ for the variance of the Parzen window kernel is relevant.

Once we compute the model spectra $P_{r}^{\prime}(f)$ and $P_{b}^{\prime}(f)$ of the ridge and boundary temperature functions respectively, we multiply them to obtain the combined model spectrum $P_{r b}^{\prime}(f)$ (see Fig. (4). Then, we find the frequency $f_{n}$ for which the model spectrum $P_{r b}^{\prime}$ assumes its maximum amplitude. We consider $f_{n}$ the mean pulse frequency of the subject during the extended time period $T$. In fact, we represent this mean pulse frequency as a Normal distribution with mean $\mu_{l}=f_{n}$ and variance $\sigma_{l}^{2}=\overline{\sigma_{n}^{2}}=0.1$.

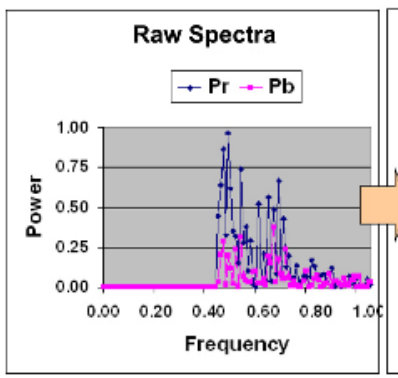

(a)

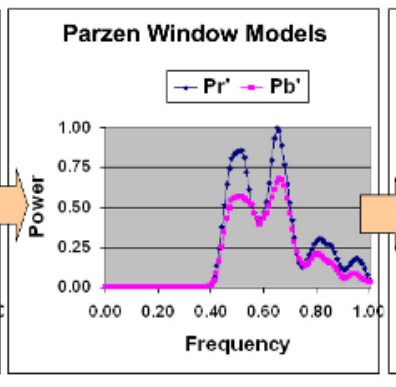

(b)

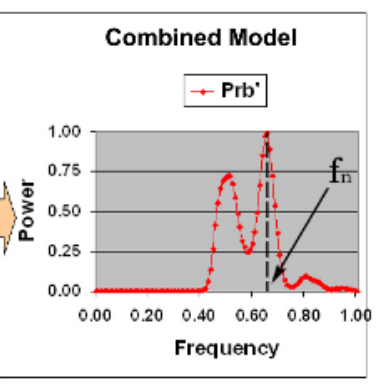

(c)

Fig. 4. All graphs are normalized: (a) Raw ridge and boundary spectra. (b) Multinormal models after the application of Parzen window. (c) Combined multi-normal model.

\subsection{Computation of the Instantaneous Pulse Frequency}

The time window of the pulse computation may vary between the period that is required for a single heartbeat (lower limit) to the long observation period we use for the mean pulse computation (upper limit). The typical period required for the completion of a heartbeat is $\sim 1 \mathrm{sec}$, although this may vary depending on the physical condition of the subject. Our imaging system operates with an average 
speed of $30 \mathrm{fps}$, so $1 \mathrm{sec}$ equates to $\sim 30$ frames. We select the time window for instantaneous pulse computation to be 512 frames $(\sim 14 \mathrm{sec})$ respectively. The chosen number is a power of 2 and facilitates the FFT computation. It is also a reasonable compromise between very long $(T \geq 30 \mathrm{sec})$ and very short $(T \sim 1 \mathrm{sec})$ observation periods.

In order to compute the combined model spectrum $P_{r b-i}^{\prime}(f)$ for the short observation period $T_{i}$, we apply exactly the same procedure that we described in section 2.3 for long observation periods. Then, we filter $P_{r b-i}^{\prime}(f)$ by multiplying it with the normal distribution $N\left(\mu_{l}, \sigma_{l}^{2}\right)$ of the mean pulse:

$$
P_{r b-i}^{\prime \prime}(f)=P_{r b-i}^{\prime}(f) * N\left(\mu_{l}, \sigma_{l}^{2}\right) .
$$

In essence, we use the mean pulse frequency to localize our attention in the instantaneous pulse frequency spectrum. Then, we compute the frequency $f_{i}$ for which the amplitude of the spectrum $P_{r b-i}^{\prime \prime}(f)$ is maximum. This is the tentative instantaneous pulse frequency.

\subsection{Post-processing}

The instantaneous pulse frequency computation described in section 2.4 may occasionally be affected by noise despite the defensive mechanisms built into the methodology. To address this problem we use an estimation function that takes into account the current measurement as well as a series of past measurements. This way, abrupt isolated measurements are smoothed over by the natural continuity constraint.

The instantaneous pulse frequency computation is being performed over the previous $T_{i}$ frames $\left(T_{i}=512\right)$. We convolve the current power spectrum $P_{c}=$ $P_{r b-i}^{\prime \prime}$ with a weighted average of the power spectra computed during the previous $M$ time steps (see Fig [5]). We chose $M=60$, since at the average speed of $30 \mathrm{fps}$ sustained by our system, there is at least one full pulse cycle contained within 60 frames even in extreme physiological scenarios. Therefore, the historical contribution of our estimation function remains meaningful at all times.

Specifically, the historical frequency response at a particular frequency $f$ is given as the summation of all the corresponding frequency responses for the $M$ spectra, normalized over the total sum of all the frequency responses for all the historical $M$ spectra:

$$
H(f)=\frac{\sum_{c=1}^{M} \overline{P_{c}}(f)}{\sum_{c=1}^{M} \sum_{j=1}^{F} P_{c}(j)}
$$

Finally, we convolve the historical power spectrum $\bar{H}$ with the current power spectrum to filter out transient features. We then designate as pulse the frequency $f_{\text {pulse }}$ that corresponds to the highest energy value of the filtered spectrum within the operational frequency band (see Fig 5 ). 


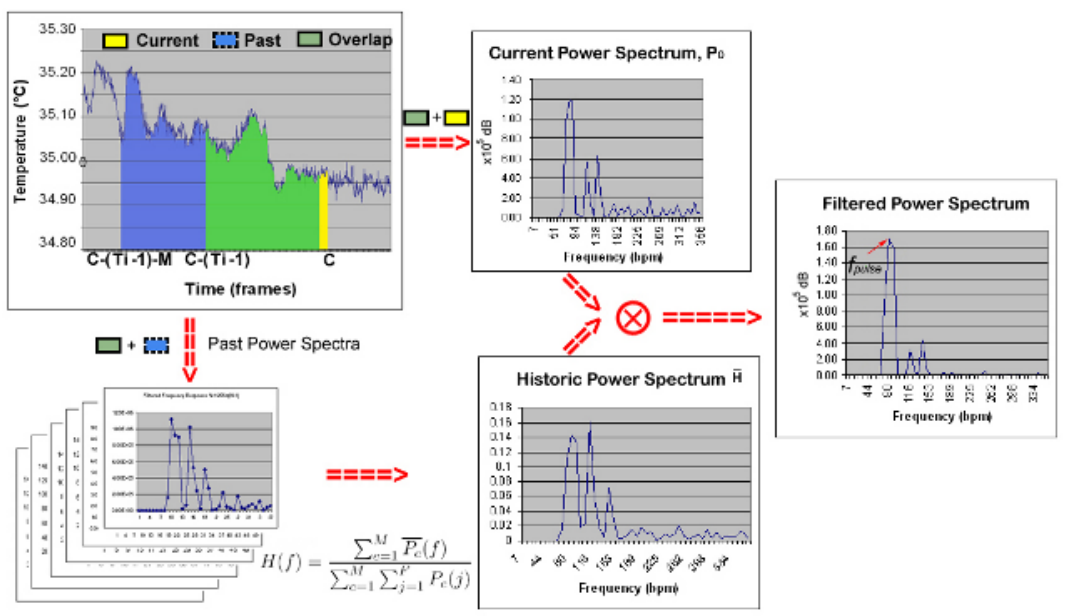

Fig. 5. Estimation Function

\section{Experimentation and Discussion}

We used a high quality Thermal Imaging (TI) system for data collection. The centerpiece of the TI system is a Phoenix InSb $640 \times 480$ Mid-Wave Infrared (MWIR) camera [10]. We recorded 34 thermal clips from the faces of 34 subjects while resting in an armchair. Concomitantly we recorded ground-truth pulse signals with the ML750 PowerLab/4SP [1] data acquisition system, accessorised with a piezo-electric sensor. The data set features subjects of both genders (24 males vs. 10 females), different races, and with varying physical characteristics. All imaging measurements were performed on a major facial vessel, that is, carotid, temporal, or supra-orbital.

We evaluated the performance of the method regarding the mean pulse computation by calculating the accuracy against the mean ground-truth measurements. We evaluated the performance of the method regarding the instantaneous pulse computation by calculating the cumulative sums (CuSum) between the instantaneous imaging measurements and their corresponding ground-truth ones.

The overall accuracy of the mean pulse measurement using the new method has improved to $92.1 \%$, compared to the previous method's [1 $88.5 \%$ performance. The new method improved dramatically the accuracy for 21 subjects that have clear thermal vessel imprints (from $88.5 \%$ to $96.9 \%$ ). These are typically the cases of lean subjects where the vessel is not obstructed by a thick fat deposit. The stellar performance is due to accurate localization of the boundary signal, which weighs heavily in the current method. Further improvements in the quantification of the boundary signal under difficult conditions is the focus of our ongoing efforts.

The overall CuSum error is only $7.8 \%$, which indicates a strong performance in instantaneous pulse measurements. 


\section{Acknowledgment}

This material is based upon work supported by the National Science Foundation under Grant No. 0414754, entitled "Interacting with Human Physiology." Any opinions, findings, and conclusions or recommendations expressed in this material are those of the author(s) and do not necessarily reflect the views of the National Science Foundation.

\section{References}

[1] Sun, N., Garbey, M., Merla, A., Pavlidis, I.: Imaging the cardiovascular pulse. In: Proceedings of the 2005 IEEE Computer Society Conference on Computer Vision and Pattern Recognition. Volume 2., San Diego, California, USA (2005) 416-21

[2] Murthy, R., Pavlidis, I., Tsiamyrtzis, P.: Touchless monitoring of breathing function. In: Proceedings of the 26th Annual International Conference of the IEEE Engineering in Medicine and Biology. Volume 2., San Francisco, California (2004) 1196-9

[3] Fei, J., Zhu, Z., Pavlidis, I.: Imaging breathing rate in the $\mathrm{CO}_{2}$ absorption band. In: Proceedings of the 27th Annual International Conference of the IEEE Engineering in Medicine and Biology, Shanghai, China (2005) 700-5

[4] Garbey, M., Merla, A., Pavlidis, I.: Estimation of blood flow speed and vessel location from thermal video. In: Proceedings of the 2004 IEEE Computer Society Conference on Computer Vision and Pattern Recognition. Volume 1., Washington D.C. (2004) 356-63

[5] Pavlidis, I., Levine, J.: Monitoring of periorbital blood flow rate through thermal image analysis and its application to polygraph testing. In: Proceedings of the 23rd Annual International Conference of the IEEE Engineering in Medicine and Biology. Volume 3., Istanbul,Turkey (2001) 2826-9

[6] Press, W.H., Teukolsky, S.A., Vetterling, W.T., Flannery, B.P.: 12. In: Numerical Recipes in C. 2nd edn. Cambridge University Press, New York, New York (1992) $504-21$

[7] Arfken, G.: 14. In: Fourier Series. 3 edn. Academic Press, Orlando, Florida (1985) 760-93

[8] Tsiamyrtzis, P., Dowdall, J., Shastri, D., Pavlidis, I., Frank, M., Ekman, P.: Lie detection - recovery of the periorbital signal through tandem tracking and noise suppression in thermal facial video. In Carapezza, E.M., ed.: Proceedings of SPIE Sensors, and Command, Control, Communications, and Intelligence (C3I) Technologies for Homeland Security and Homeland Defense IV. Volume 5778., Orlando, Florida (2005)

[9] Duda, R.O., Hart, P.E., Stork, D.G. In: Pattern Classification. A WileyInterscience Publication, New York, New York (2001)

[10] Indigo Systems Inc. 70 Castilian Dr., Goleta, California 93117-3027: (http://www. indigosystems.com)

[11] ADInstruments Pty Ltd Unit 6, 4 Gladstone Rd, Castle Hill, NSW 2154, Australia: PowerLab ADInstruments Owners Manual. (2004) 